\title{
(C) OPEN ACCESS \\ Promoting engagement by patients and families to reduce adverse events in acute care settings: a systematic review
}

\author{
Zackary Berger, ${ }^{1,2}$ Tabor E Flickinger, ${ }^{3}$ Elizabeth Pfoh, ${ }^{4}$ \\ Kathryn A Martinez, ${ }^{4,5}$ Sydney M Dy ${ }^{4}$
}

- Additional material is published online only. To view please visit the journal online (http://dx.doi.org/10.1136/bmjqs2012-001769).

For numbered affiliations see end of article.

\section{Correspondence to}

Dr Zackary D Berger, Division of General Internal Medicine, Department of Medicine, The Johns Hopkins Outpatient Center, 601 North Caroline Street, Suite 7143, Baltimore, MD 21287-0941, USA; zberger1@jhmi.edu

Received 18 December 2012 Revised 18 November 2013 Accepted 22 November 2013 Published Online First 13 December 2013

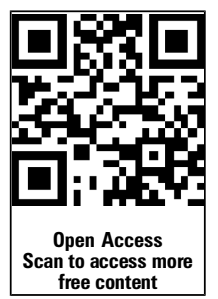

CrossMark

To cite: Berger $Z$, Flickinger TE, Pfoh $\mathrm{E}$, et al. BMJ Qual Saf 2014;23: 548-555.

\begin{abstract}
Introduction Patient-centeredness is central to healthcare. Hospitals should address patients' unique needs to improve safety and quality. Patient engagement in healthcare, which may help prevent adverse events, can be approached as an independent patient safety practice (PSP) or as part of a multifactorial PSP.
\end{abstract}

\section{Objectives This review examines how} interventions encouraging this engagement have been implemented in controlled trials.

Methods We searched Medline, CINAHL, Embase and Cochrane from 2000 to 2012 for English language studies in hospital settings with prospective controlled designs, addressing the effectiveness or implementation of patient/family engagement in PSPs. We separately reviewed interventions implemented as part of selected broader PSPs by way of example: hand hygiene, ventilator-associated pneumonia, rapid response systems and care transitions.

Results Six articles met the inclusion criteria for effectiveness with a primary focus on patient engagement. We identified 12 studies implementing patient engagement as an aspect of selected broader PSPs. A number of studies relied on patients' possible function as a reporter of error to healthcare workers and patients as a source of reminders regarding safety behaviours, while others relied on direct activation of patients or families. Definitions of patient and family engagement were lacking, as well as evidence regarding the types of patients who might feel comfortable engaging with providers, and in what contexts.

Conclusions While patient engagement in safety is appealing, there is insufficient highquality evidence informing real-world implementation. Further work should evaluate the effectiveness of interventions on patient and family engagement and clarify the added benefit of incorporating engagement in multifaceted approaches to improve patient safety endpoints. In addition, strategies to assess and overcome barriers to patients' willingness to actively engage in their care should be investigated.

\section{INTRODUCTION}

\section{How important is the problem?}

Patient-centeredness is a central aspect of healthcare, including hospital care. Patients have unique needs that hospitals should address to improve safety and quality. ${ }^{1}$ These unique needs can be addressed more fully by encouraging patient and family engagement in safety, making use of patients' knowledge and unique concerns connected to their healthcare, bringing their own perspective and agendas to bear. ${ }^{2}$ Such involvement is promoted by several international organisations, and a variety of educational materials have been developed to facilitate patient engagement in patient safety practices (PSPs). ${ }^{3}$

\section{What is patient safety practice (PSP)?}

Engagement can be seen as an 'umbrella' term incorporating various approaches rather than a specific process, team or technology. Generally, definitions centre on patient/family participation in care from the viewpoint of respect for patients or families as persons, consumer rights or care coordination to encourage patients to be active in preventing or reporting safety issues. 45

Patient and family engagement can be understood as a PSP in various ways. First, patient engagement can be approached as an overarching philosophy applicable to a number of PSPs. For example, in a 
reflection on the extent of progress in the first 10 years of widespread efforts to improve patient safety, Wachter added patient engagement as a key domain of patient safety which includes patient involvement in error prevention and disclosure of errors to patients, while acknowledging its 'unproven' nature owing to a paucity of evidence. ${ }^{6}$ Second, increasing patient engagement can be a specific component of patient safety interventions.

While few PSPs are implemented with the primary goal of patient/family engagement, improving engagement can be a core component of broader PSP interventions. For example, a PSP such as the implementation of a Rapid Response System (RRS) may have a primary focus on provider reporting, but also include a mechanism for patients or families to report concerns about their clinical status to the RRS. However, because patient engagement is often not the primary target of broader PSP interventions, empirically measured changes in patient engagement after implementation may not be reported. Alternatively, patient engagement may be treated as a contextual variable that moderates the effectiveness of the PSP and, as a result, may not be separately reported.

This systematic review focuses on the definition and implementation of patient engagement as part of selected hospital-based PSPs with the primary intent of increasing patient/family involvement to improve patient safety. The extensive literature on patient engagement from a theoretical or advocacy perspective, observational studies and studies on patient engagement not directly related to PSPs (eg, health literacy, improving patient adherence to medications) has been addressed in a number of other reviews. ${ }^{7-13}$

\section{Why should PSPs work?}

Patients are a central part of the treatment team who may be aware of errors not obvious to their providers. ${ }^{2}$ As a result, patients are uniquely positioned to provide important information potentially unavailable from other sources such as medical records. ${ }^{2}$ Previous literature has demonstrated that patients are highly motivated to decrease medical risk and improve outcomes ${ }^{14}$ but a number of barriers often prevent patients from fully engaging in safety practices. A recent systematic review ${ }^{14}$ of facilitators and barriers affecting patients' ability and willingness to participate in initiatives to reduce medical errors found that illness severity and the self-perception of being subordinate to physicians were associated with reluctance by patients to participate in safety practices. Alternatively, key facilitators to patient involvement in safety practices include patients' cognitive characteristics, the doctor-patient relationship and organisational factors of the healthcare system. Finally, since many safety problems occur at the bedside and can be observed and potentially prevented by patients, they are both an important source of information on potentially avoidable adverse events and a mechanism by which, through their involvement, they can improve safety. ${ }^{7}$ Our own conceptual model understanding the potential impact of patient and family engagement on outcomes is shown in supplemental figure 1 in the online appendix.

\section{What are the beneficial effects of PSP?}

The most recent systematic review of patient engagement in safety covered articles published through October 2008. ${ }^{8}$ To evaluate how patient engagement is implemented, we performed a systematic review of Medline, CINAHL, Embase and Cochrane from 2000 to 2012 using synonyms for patient engagement and patient safety including physician-patient relations, patient participation and patient-centred care (see search strategy in online appendix) to retrieve studies of independent interventions meant to improve patient or family engagement. We also separately reviewed patient/family engagement interventions implemented as part of selected broader PSPs including hand hygiene, ventilator-associated pneumonia (VAP) and RRS. In addition, in order to understand how the concept of patient and family engagement in safety can be applied to the PSP of care transitions, we examined the most widely published intervention, the Care Transitions Intervention (CTI).

We addressed these four PSPs as illustrative examples of the role of engagement in pre-existing practices. A number of other PSPs have recently been reviewed elsewhere in the literature using similar methodology, ${ }^{15-17}$ and a complete examination of the part of patient and family engagement in every extant PSP would be unfeasible. This review was part of a larger report on the evidence for PSPs. ${ }^{18}$

Only English language studies from the USA, UK, Canada and Australia were included owing to potentially significantly different cultural issues regarding patient engagement in other countries as well as potential differences in tools for promoting engagement. Our review was limited to studies in hospital settings because patient engagement in the home setting would be difficult to differentiate from patient self-management of medications and care when providers are not present. Finally, only systematic reviews and prospective controlled studies were included (see supplemental figure 2 in online appendix).

For the purposes of our review, we classified the included studies in two ways: (1) as an independent PSP which sought to directly encourage patient/family engagement in safety practices; or (2) as a domain of a pre-existing PSP in which patient/family engagement was a component. We also separately examined the literature regarding CTIs, a broader set of patient safety interventions unlike the pre-existing PSPs with specific patient safety targets. Given the paucity of studies addressing effectiveness and the difficulty of assessing the effectiveness of patient/family engagement when 
part of a pre-existing PSP, we focus here on describing the characteristics of the interventions.

\section{PRIMARY PATIENT ENGAGEMENT INTERVENTIONS: SYSTEMATIC REVIEW}

The literature search yielded 4560 potentially relevant unique articles; 44492 were excluded during abstract screening, leaving 68 for full article review. Of these 68, 49 were excluded, leaving 19 articles for full review. Six articles met the inclusion criteria for studies of patient or family engagement as an independent PSP. ${ }^{19-24}$

Of the six included studies, four of these focused on improving patient engagement centred around healthcare worker $(\mathrm{HCW})$ hand hygiene $(\mathrm{HH})$ practices. The interventions included: (1) encouraging patients to ask all HCWs with direct contact if they had washed/sanitised their hands ${ }^{19}$; (2) putting up posters to encourage patients to ask providers to wash their hands ${ }^{20} 21$; (3) watching the Pink Patient Safety video $^{21}{ }^{22}$; (4) a visit by a premedical student to discuss $\mathrm{HH}$ with the patient ${ }^{19}$; and (5) application of other patient-directed visual aids. ${ }^{20}$ One of these studies involved multiple components ${ }^{19}$ while several were single component. ${ }^{20-23}$

All four studies that focused on $\mathrm{HH}$ used pre-post methodology. The use of a multifaceted intervention by McGuckin et $a l^{19}$ resulted in patients asking physicians about $\mathrm{HH} 40 \%$ of the time and nurses $95 \%$ of the time. Stone $e t a l^{20}$ found that, following the intervention, median use of alcohol hand rub increased statistically significantly but the rate of hospitalacquired infections did not change. In 2011, Davis et $a l^{21}$ found that more patients who saw the Pink Safety video reported they would be comfortable asking about hand washing and notifying healthcare providers about medication errors. A subsequent 2012 study ${ }^{22}$ found that patients reported increased willingness to ask HCWs about hand washing and with help with their own hygiene, and expressed an increased appreciation of the importance in participating in safety-related behaviours following the intervention.

However, despite the intervention's positive effect on patient appreciation for the importance of participation in safety practices, the patients' perceived importance of these behaviours remained greater than their willingness to participate in them. Moreover, while the use of a multifaceted intervention about the importance of $\mathrm{HH}$ increased patient willingness to ask nurses about $\mathrm{HH}$ practices, willingness to interact with doctors about $\mathrm{HH}$ was still quite low. ${ }^{20}$

The only randomised controlled study included in our review ${ }^{24}$ was part of a multicomponent intervention providing 209 patients with a personalised medication list in order to reduce the incidence of adverse drug events (ADEs) and close calls in a teaching hospital without computerised physician order entry. Both intervention and control groups were provided with general education about drug safety and the intervention group was also provided with a list of their medications. No significant difference in ADEs or close calls was observed between the two groups at follow-up.

We identified one relevant systematic review of patient and family engagement in safety. ${ }^{25}$ The authors of the review found limited evidence, of poor quality, for benefits of patient involvement in patient safety; the reviewed studies mostly involved patient medication management. ${ }^{8} 2426$

Because of the small number of studies meeting the criteria and the heterogeneity of their design and their outcomes, we could not perform evidence grading.

\section{How has the PSP been implemented and in what contexts?}

We identified 12 examples of patient/family engagement as part of selected other broader PSPs. These included studies focused on HH, RRS, surgical checklists, prevention of falls, prevention of VAP, prevention of medical errors after discharge and CTIs (see table in online appendix). Each PSP used slightly different strategies to engage patients but, on the whole, patients and their families were encouraged to take part in ensuring their own safety. We discuss separately the CTI, in which patient engagement is part of a multifaceted intervention.

Patient engagement in implementation of hand hygiene $(\mathrm{HH})$ interventions

Three papers highlighted the barriers and facilitators associated with implementing patient engagement strategies as part of $\mathrm{HH}$ interventions. In the 2011 review by McGuckin et $a l^{27}$ the term 'patient empowerment' is used instead of 'patient engagement', but the terms can be understood as referring to similar phenomena. The review found that while 80$90 \%$ of patients reported willingness to ask HCWs to wash their hands, only $60-70 \%$ of patients complied, suggesting that, while patients are theoretically willing to ask HCWs, barriers exist which make the actual request difficult, at least on some occasions. McGuckin et $a l^{28}$ also addressed patient empowerment as an approach to motivating strategies in $\mathrm{HH}$ interventions. The authors identified several barriers to patient engagement, emphasising the negative social reaction that patients might feel when asking providers about $\mathrm{HH}$.

A more recent review by Landers et $a l^{29}$ summarised patient factors that have been found to be associated with patient willingness to encourage HCWs to perform hand washing. These factors included extroverted patient personality, patient belief that they could control the HCW's behaviour, younger age of patient, an awareness of the severity of healthcare-associated infections and an invitation by the $\mathrm{HCW}$ to discuss $\mathrm{HH}$. Conversely, factors that 
predisposed against this willingness included older age and trust that the HCW would perform hand washing. ${ }^{29}$

Patient engagement in prevention of healthcare-associated infection We found only one study that was aimed at improving patient engagement associated with the prevention of healthcare-associated infection. Similar to the $\mathrm{HH}$ interventions, Hart et $a l^{30}$ used a poster in patients' rooms as an intervention to address potential safety lapses in five domains for the prevention of healthcare-associated infection including $\mathrm{HH}$. In this study, patient engagement was conceptualised by patient empowerment to address safety lapses regarding infection prevention that were addressed by the poster. The intervention was facilitated by reinforcement of the goal of the poster by other elements of the intervention.

Patient engagement in implementation of RRS interventions

Three studies were found that included implementation of patient engagement strategies as part of RRS interventions. All three interventions ${ }^{31-33}$ encouraged the family to call the rapid response team themselves. Cited barriers for the RRS interventions included: physicians concerned that their role would be undermined $^{32}$ and that resources would be overwhelmed, and endorsement of hospital administration, physicians $^{33}$ and staff. Facilitators included leadership and physician involvement ${ }^{32} 33$ and providers' understanding of RRS as an extension of care they already provided. $^{32}$ Ray et $a l^{31}$ implemented a paediatric RRS based on direct family activation to 'empower family members to seek help when serious concerns arise'. The 'direct family activation' was a direct telephone number to reach the RRS, which families could reach from any room in the hospital. In addition, families were educated via posters in patients' rooms and flyers. Nurses were trained in explaining the RRS activation to families and were given reminders in the electronic medical record to solicit feedback on levels of family awareness of the RRS at regular intervals.

Dean et $a l^{32}$ described a similar early warning system that was aimed at empowering patients and families to participate in PSPs by integrating them into the RRS protocols of a major children's hospital. Conditions under which patients/families were encouraged to call the RRS included: a noticeable medical change that had been unaddressed; a breakdown in care or uncertainty regarding treatment; the administration of a medication that causes an adverse effect or that the patient/family believed had not been sufficiently explained; or a treatment that the patient/ family believed was meant for another patient or contravened their doctor's wishes. This study did not detail how patients/families were empowered or educated to overcome barriers to using the system.

Gerdik et $a l^{33}$ studied implementation of a patient/ family-activated RRS. Picker's 'Eight Dimensions of
Patient-Centred Care' provided the conceptual framework, which emphasises involvement of family and friends in decision-making. Implementation of the intervention involved distribution of written educational materials, informational signs, instructional labels for telephones and scripted education and training by staff. Satisfaction with the RRS programme among patients and families was high.

Patient engagement in implementation of falls interventions

We found two studies aimed at increasing patient engagement in preventing falls. Both studies used nurses to engage patients in fall prevention by engaging with them and families directly to educate and encourage specific behaviours. Barriers cited included staff turnover, high patient-to-nurse ratios, high patient turnover or high patient volume, competing demands on nursing staff, lack of buy-in from staff $^{34}$ and intervention complexity. ${ }^{35}$

Krauss et $a^{34}$ implemented an educational intervention to reduce patient falls according to a quasi-experimental design among nursing staff, nursing secretaries and patient care technicians in an academic hospital. While patient or family engagement was not mentioned specifically as part of the implementation or its conceptual background, nurses were directed to educate all patients in fall prevention. For patients with a high risk of falling, nursing staff were instructed to reinforce falls prevention education with both patients and family. Staff received feedback on their unit's fall rates during the implementation via meetings and flyers. Nursing staff's knowledge and use of prevention strategies improved.

van Gaal et $a l^{35}$ implemented a multicomponent intervention to reduce the risk of pressure ulcers, falls and urinary tract infections in 10 wards in four hospitals and 10 wards in six nursing homes. Patient involvement was used as part of the intervention with the goal of enhancing the usefulness of the multicomponent intervention overall. Oral and written information was given to patients at risk of specific ADEs. Implementation on every participating ward included educational meetings for nurses and informational brochures for patients at risk of any one of the adverse events addressed.

Patient engagement in implementation of surgical checklist interventions

Most studies of inventions to prevent wrong site surgery have focused on checklists for surgeons or anaesthesiologists to perform before surgery. ${ }^{36-38}$ Although patient interaction may be part of the checklist, such as verbally verifying patient identity and surgical site, ${ }^{37}$ the provider team is normally the target of such interventions. We identified two studies examining patient engagement as a means to avoid wrong site surgery. One study, in a private foot and ankle practice, gave patients written instructions to mark the limb not to be operated on with the label 'NO'. 
Observed patient compliance was 59\%. ${ }^{39}$ The second study, in a university-affiliated orthopaedic practice, gave patients both verbal and written instructions to mark the intended surgical site with the label 'YES' and provided a marking pen to do so. ${ }^{40}$ Patient compliance in this study was $68.2 \%$, with higher compliance in patients whose primary language was English and whose surgery occurred sooner after instructions were given. Barriers included non-English primary language, cultural tendency to rely on physicians, younger patient age and time between study enrolment and surgery. ${ }^{40}$

\section{Family engagement in preventing VAP}

We identified one study ${ }^{41}$ which developed, implemented and monitored the success of a prevention initiative for VAP which included family education. Families were provided with information about how they could help with VAP prevention. A survey administered postintervention indicated that families thought this education improved their communication with providers.

\section{Patient engagement in implementation of CTls}

Patient engagement in transitional care is an important example of encouraging patient activation as a component of a larger multifaceted approach to safely move patients out of an acute care setting, unlike the specific PSPs discussed above which focus on specific patient safety targets. Pre-discharge interventions may include patient engagement through patient and/or caregiver education. Post-discharge interventions may include outreach to patients and/or caregivers by means of follow-up phone calls or other methods. 'Bridging' interventions may include a combination of these components.

As an example, the most widely published intervention, the CTI, has been implemented and evaluated in multiple settings. ${ }^{42-46}$ The CTI is designed to provide patients and caregivers with the tools and skills to take a more active role in their care. It is based on three pillars of medication self-management: a patient-centred record, follow-up and identification of 'red flags' with instructions on how to respond to them. In this study, patients and caregivers received in-hospital visits, telephone calls, home visits, encouragement to take an active role in care and guidance from a 'transition coach'.

Implementation issues included the training and time commitment of transition coaches and the challenge of recruiting and retaining patients in the intervention. ${ }^{47}$ Mean patient activation scores were moderately higher for sites with full sustainability plans than for sites with partial or minor plans, suggesting that greater engagement in the programme at the site level could affect engagement by patients receiving the intervention. ${ }^{47}$ Qualitative data indicated that patients' perception of a caring relationship with transition coaches fostered greater patient engagement in the programme. ${ }^{46}$

\section{Are there any data about costs?}

None of the reviewed studies directly evaluated the costs or cost-effectiveness of practices designed to promote patient or family engagement with safety.

\section{Are there any data about the effect of context on} effectiveness?

As noted above, McGuckin et al ${ }^{19}$ recorded the frequency with which patients asked various members of the care team about their HH practices; however, this outcome was not linked to any specific hypotheses about the impact of context on effectiveness. Generally, the limited nature of the evidence presented here precludes any conclusions about effectiveness.

\section{What are the harms of PSP?}

None of the included studies evaluated harms of interventions or surveys. Interventions to increase patient engagement, such as reminding HCWs to wash their hands, could theoretically adversely affect providerpatient relationships and patients' trust in providers. Patients might fear adverse consequences, or healthcare providers could become overly reliant on patient engagement and more lax in their own safety practices. In addition, the ability or interest of patients to become engaged in patient safety might significantly vary among individuals and families, who might feel guilty were errors to occur supposedly 'on their watch'. ${ }^{-9}$

\section{DISCUSSION}

Patient and family engagement is an emerging area in patient safety research, with few published effectiveness studies. However, patient engagement is an increasingly important component of patient safety initiatives in hospitals. We identified six studies which addressed an independent intervention for patient/ family engagement in safety that was not part of a larger PSP. Moreover, a number of recent studies have described successes and challenges of implementing patient engagement as part of single and multicomponent patient safety interventions. In some studies, patient and family engagement is pursued via education and providing information about the PSP. ${ }^{28}{ }^{31} \mathrm{In}$ others, engagement is encouraged via directions to take a specific action in response to a particular type of situation ${ }^{32-34}$ while still others involve operationalised engagement as a patient or family member's willingness to interact with members of the healthcare team to promote a PSP such as HH. ${ }^{31} 32$

In this review we focused on implementation of interventions to promote patient and family engagement and not on the effectiveness of these implementations, given the paucity and heterogeneity of the data. In four studies involving PSPs in hospital-acquired infection prevention, surgical checklists and $\mathrm{HH}$, patients and families were encouraged to directly address HCWs to point out lapses or remind them of safety behaviours. ${ }^{28} 314041$ This 
approach to patient engagement is dependent on the patient or family themselves noticing safety lapses as a means of improving patient safety. ${ }^{48-51}$ This role has often been described in the safety literature as a means of preventing medical errors. The effectiveness of this approach depends on patients' willingness and ability to participate in reporting clinical errors and communicating about them with HCWs. As noted above, the patient-physician relationship can serve as a facilitator to such willingness, while patients' perceived subordination can be a barrier. ${ }^{14}$ The limited evidence regarding the effect of patient engagement on the patient-doctor relationship indicates that, while physicians might be theoretically supportive of such engagement, the effect on the relationship might not be positive. ${ }^{52}$ In this regard, an educational patient safety campaign might be of use in optimising the context for interventions requiring doctor-patient communication about safety. ${ }^{53}$

The use of direct activation was another approach to improve patient and family engagement. This was most relevant to the RRS studies, although it is also applicable to the studies on prevention of VAP and falls prevention. Organisational factors, as well as patients' willingness and ability to be activated, might contribute to this approach.

We also examined the evidence in one area, CTIs, in which patient and family engagement was one part of a multicomponent model. We focused on one particular variety of care transition intervention that has been widely implemented as an exemplar of the application of patient engagement in this area. The importance of the patient's relationship with the transition coach provides further indication of the possible importance of patient-provider relationships in fostering patient engagement as part of a PSP.

We reviewed the evidence only for selected PSPs representative of different types of PSPs. Other PSPs not addressed in this review might be relevant to patient and family engagement in safety and involve types of interventions not addressed here. For example, Kripalani et $a{ }^{54}$ in a randomised controlled trial aimed at reducing clinically important medication errors after discharge, included tailored inpatient counselling by clinical pharmacists as part of a multicomponent intervention in acute care hospitals. The intervention, which was tailored to patients with low health literacy, involved education of patients regarding their medication regimen by pharmacists. The mechanism by which patient engagement contributed to the intervention was not specified, but the pharmacist counselling addressed issues such as understanding of medications and prescription labels, barriers to adherence and social support.

Our systematic review has a number of limitations. Due to the small number of studies, the strength of evidence is, by necessity, low, and will likely improve over time with the emergence of other studies on this topic. In addition, these conclusions may not be generalisable to other populations, clinical conditions or PSPs. Finally, a culture of safety may affect both the willingness of patients to engage in their care and the receptiveness by HCWs. However, it was outside the scope of this study to assess the impact of culture on patient engagement. In addition, we were not able to discuss in this review a rich literature on the theory and advocacy of patient engagement, since most of these studies were not empirical studies of implementation. Finally, the major weakness of this review is the lack of literature assessing the effectiveness of these interventions-that is, whether these interventions actually improve patient and family engagement and safety outcomes.

Future work must address basic and applied concerns across the spectrum of conceptual foundations and experimental design including research questions that need to be answered: the definition and measurement of patient and family engagement; the safety endpoints that should be addressed; the effectiveness of these interventions both in promoting engagement and in improving patient safety outcomes; and methodological issues concerning study design.

Patient participation in safety practices may be influenced by patient self-perception, the patient-physician relationship, societal norms and the healthcare environment, including whether the organisational culture supports patients' participation. ${ }^{6-9}$ The reaction of physicians and other HCWs to patient and family engagement in safety should be examined. Also important to address in future work is the variety of approaches that have been taken to promote patient engagement, whether as an independent intervention or as part of an intervention housed within an existing PSP. Clarifying the theoretical models underlying the mechanism of patient engagement-whether direct patient activation or encouraging the patient's role as a source of information regarding error-can help motivate successful interventions on this basis.

In conclusion, while patient engagement in safety is an appealing approach, there is still little evidence regarding the particular details of implementation needed to guide future PSPs involving such engagement. Moreover, some literature suggests that patients may not necessarily feel comfortable playing such a role in their care. Much remains unknown regarding patient and family engagement in safety, including the optimum strategy of implementation and whether such an approach is in fact effective.

Author affiliations

${ }^{1}$ Division of General Internal Medicine, Johns Hopkins University School of Medicine, Baltimore, Maryland, USA

${ }^{2}$ Johns Hopkins Berman Institute of Bioethics, Baltimore, Maryland, USA

${ }^{3}$ Division of General Medicine, Geriatrics, and Palliative Care, University of Virginia School

of Medicine, Charlottesville, Virginia, USA

${ }^{4}$ Department of Health Policy and Management, Johns

Hopkins University School of Public Health, Baltimore, Maryland, USA 
${ }^{5}$ Division of General Medicine, University of Michigan Health System, Center for Clinical Management Research, Ann Arbor VA Medical Center, Ann Arbor, Michigan, USA

Acknowledgements This review is based on work reported in a technical report for the Agency for Healthcare Research and Quality. The citation for the chapter and full report appear as reference 18 in the present paper and can be accessed at http:// www.ncbi.nlm.nih.gov/books/NBK133412/

Contributors ZB, TEF, EP and KAM were responsible for the study concept, design, analysis, interpretation and preparation of the manuscript. SMD was responsible for the study concept, interpretation and preparation of the manuscript.

Funding This work was supported by funding from the Agency for Healthcare Research and Quality (AHRQ), US Department of Health and Human Services (Contract No.

HHSA-290-2007-10062I). AHRQ reviewed contract deliverables to ensure adherence to contract requirements and quality, and a copyright release was obtained from AHRQ prior to submission of this manuscript.

\section{Competing interests None.}

Provenance and peer review Not commissioned; externally peer reviewed.

Open Access This is an Open Access article distributed in accordance with the Creative Commons Attribution Non Commercial (CC BY-NC 3.0) license, which permits others to distribute, remix, adapt, build upon this work noncommercially, and license their derivative works on different terms, provided the original work is properly cited and the use is non-commercial. See: http://creativecommons.org/licenses/by$\mathrm{nc} / 3.0 /$

\section{REFERENCES}

1 The Joint Commission. Advancing effective communication, cultural competence, and patient-and family-centered care: a roadmap for hospitals. Oakbrook Terrace, IL: The Joint Commission, 2010.

2 Vincent CA, Coulter A. Patient safety: what about the patient? Qual Saf Health Care 2002;11:76-80.

3 Perneger T. The Council of Europe recommendation Rec (2006) on management of patient safety and prevention of adverse events in health care. Int J Qual Health Care 2008;20:305-7.

4 Davis RE, Jacklin R, Sevdalis N, et al. Patient involvement in patient safety: what factors influence patient participation and engagement? Health Expect 2007;10:259-67.

5 Entwistle VA. Differing perspectives on patient involvement in patient safety. Qual Saf Health Care 2007;16:82-3.

6 Wachter RM. Patient safety at ten: unmistakable progress, troubling gaps. Health Aff 2010;29:165-73.

7 Koutantji M, Davis R, Vincent C, et al. The patient's role in patient safety: engaging patients, their representatives, and health professionals. Clin Risk 2005;11:99-104.

8 Schwappach DL. Review: engaging patients as vigilant partners in safety: a systematic review. Med Care Res Rev 2010;67:119-48.

9 Longtin Y, Sax H, Leape LL, et al. Patient participation: current knowledge and applicability to patient safety. Mayo Clin Proc 2010;85:53-62.

10 Coulter A, Ellins J. Effectiveness of strategies for informing, educating, and involving patients. BMJ 2007;335:24-7.

11 Iedema R, Merrick E, Piper D, et al. Codesigning as a discursive practice in emergency health services: the architecture of deliberation. J Appl Behav Sci 2010;46:73-91.

12 Hibbard J, Peters E, Slovic P, et al. Can patients be part of the solution? Views on their role in preventing medical errors. Med Care Res Rev 2005;62:601-16.
13 Hurst I. Vigilant watching over: Mothers' actions to safeguard their premature babies in the newborn intensive care nursery.

J Perinat Neonatal Nurs 2001;15:39-57.

14 Doherty C, Stavropoulou C. Patients' willingness and ability to participate actively in the reduction of clinical errors: a systematic literature review. Soc Sci Med 2012;75:257-63.

15 Shekelle PG, Pronovost PJ, Wachter RM, et al. The top patient safety strategies that can be encouraged for adoption now. Ann Intern Med 2013;158(5 Part 2):365-8.

16 Rennke S, Nguyen OK, Shoeb MH, et al. Hospital-initiated transitional care interventions as a patient safety strategy: a systematic review. Ann Intern Med 2013;158 (5 Part 2):433-40.

17 Weaver SJ, Lubomski LH, Wilson RF, et al. Promoting a culture of safety as a patient safety strategy: a systematic review. Ann Intern Med 2013;158(5 Part 2):369-74.

18 Shekelle PG, Wachter R, Pronovost P, et al. Promoting engagement by patients and families to reduce adverse events (chapter 32). In: Shekelle PG, Wachter R, Pronovost P, et al. eds. Making health care safer II: an updated critical analysis of the evidence for patient safety practices. Comparative effectiveness review No. 211. AHRQ Publication No.13-E001-EF. Rockville, MD: Agency for Healthcare Research and Quality, 2013;325-50.

19 McGuckin M, Taylor A, Martin V, et al. Evaluation of a patient education model for increasing hand hygiene compliance in an inpatient rehabilitation unit. Am J Infect Control 2004;32:235-8.

20 Stone S, Slade R, Fuller C, et al. Early communication: does a national campaign to improve hand hygiene in the NHS work? Initial English and Welsh experience from the NOSEC study (National Observational Study to Evaluate the CleanYourHandsCampaign). J Hosp Infect 2007;66:293-6.

21 Davis RE, Sevdalis N, Pinto A, et al. Patients' attitudes towards patient involvement in safety interventions: results of two exploratory studies. Health Expect 2013;16:e164-76.

22 Davis RE, Pinto A, Sevdalis N, et al. Patients' and health care professionals' attitudes towards the PINK patient safety video. J Eval Clin Pract 2012;18:848-53.

23 Fennell E. The PINK Patient Safety People. http://www. institute.nhs.uk/index.php?option $=$ com mtree\&task=viewlink\&link_id $=4506 \&$ Itemid $=2923$ (accessed 29 Mar 2013).

24 Weingart SN, Toth M, Eneman J, et al. Lessons from a patient partnership intervention to prevent adverse drug events. Int J Qual Health Care 2004;16:499-507.

25 Hall J, Peat M, Birks Y, et al. Effectiveness of interventions designed to promote patient involvement to enhance safety: a systematic review. Qual Safe Health Care 2010;19:e10.

26 Peat M, Entwistle V, Hall J, et al. Scoping review and approach to appraisal of interventions intended to involve patients in patient safety. J Health Serv Res Policy 2010;15(Suppl 1):17-25.

27 McGuckin M, Storr J, Longtin Y, et al. Patient empowerment and multimodal hand hygiene promotion: a win-win strategy. Am J Med Qual 2011;26:10-17.

28 McGuckin M, Waterman R, Storr IJ,, et al. Evaluation of a patient-empowering hand hygiene programme in the UK. $J$ Hosp Infect 2001;48:222-7.

29 Landers T, Abusalem S, Coty MB, et al. Patient-centered hand hygiene: the next step in infection prevention. Am J Infect Control 2012;40(4 Suppl1):S11-17. 
30 Hart R. The effects of a poster in informing and empowering patients in infection prevention and control. J Infec Prev 2012;13:146-53.

31 Ray EM, Smith R, Massie S, et al. Family alert: implementing direct family activation of a pediatric rapid response team. Jt Comm J Qual Patient Saf 2009;35:575-80.

32 Dean BS, Decker MJ, Hupp D, et al. Condition HELP: a pediatric rapid response team triggered by patients and parents. J Healthc Qual 2008;30:28-31.

33 Gerdik C, Vallish RO, Miles K, et al. Successful implementation of a family and patient activated rapid response team in an adult level 1 trauma center. Resuscitation 2010;81:1676-81.

34 Krauss MJ, Tutlam N, Costantinou E, et al. Intervention to prevent falls on the medical service in a teaching hospital. Infect Control Hosp Epidemiol 2008;29:539-45.

35 van Gaal BG, Schoonhoven L, Mintjes JA, et al. Fewer adverse events as a result of the SAFE or SORRY? programme in hospitals and nursing homes. Part I: Primary outcome of a cluster randomised trial. Int J Nurs Stud 2011;48:1040-8.

36 Garnerin P, Ares M, Huchet A, et al. Verifying patient identity and site of surgery: improving compliance with protocol by audit and feedback. Qual Saf Health Care 2008;17:454-8.

37 Nilsson L, Lindberget O, Gupta A, et al. Implementing a pre-operative checklist to increase patient safety: a 1-year follow-up of personnel attitudes. Acta Anaesthesiol Scand 2010;54:176-82.

38 Owers C, Lees E, Roberts J. Correct site surgery-are we up to standard? Cent Eur J Med 2010;5:407-10.

39 Bergal LM, Schwarzkopf R, Walsh M, et al. Patient participation in surgical site marking: can this be an additional tool to help avoid wrong-site surgery? J Patient Saf 2010;6:221-5.

40 DiGiovanni CW, Kang L, Manuel J. Patient compliance in avoiding wrong-site surgery. J Bone Joint Surg Am 2003;85-A:815-19.

41 DeJuilio PA, Rivera SJ, Huml JP. A successful VAP prevention program. J Respir Care Pract 2012;25:26.

42 Coleman EA, Smith JD, Frank JC, et al. Preparing patients and caregivers to participate in care delivered across settings: the Care Transitions Intervention. J Am Geriatr Soc 2004;52:1817-25.
43 Coleman EA, Parry C, Chalmers SA, et al. The central role of performance measurement in improving the quality of transitional care. Home Health Care Serv Q 2007;26:93-104.

44 Parry C, Min SJ, Chugh A, et al. Further application of the care transitions intervention: results of a randomized controlled trial conducted in a fee-for-service setting. Home Health Care Serv Q 2009;28:84-99.

45 Voss R, Gardner R, Baier R, et al. The care transitions intervention: translating from efficacy to effectiveness. Arch Intern Med 2011;171:1232-7.

46 Parrish MM, O’Malley K, Adams RI, et al. Implementation of the care transitions intervention: sustainability and lessons learned. Prof Case Manag 2009;14:282-93.

47 Parry C, Kramer HM, Coleman EA. A qualitative exploration of a patient-centered coaching intervention to improve care transitions in chronically ill older adults. Home Health Care Serv Q 2006;25:39-53.

48 Weissman JS, Schneider EC, Weingart SN, et al. Comparing patient-reported hospital adverse events with medical record review: do patients know something that hospitals do not? Ann Intern Med 2008;149:100-8.

49 Taylor BB, Marcantonio ER, Pagovich O, et al. Do medical inpatients who report poor service quality experience more adverse events and medical errors? Med Care 2008;46:224-8.

50 Weingart SN, Pagovich O, Sands DZ, et al. Patient-reported service quality on a medicine unit. Int J Qual Health Care 2006;18:95-101.

51 Weingart SN, Pagovich O, Sands DZ, et al. What can hospitalized patients tell us about adverse events? Learning from patient-reported incidents. J Gen Intern Med 2005;20:830-6.

52 Schwappach DL, Frank O, Davis RE. A vignette study to examine health care professionals' attitudes towards patient involvement in error prevention. J Eval Clin Pract 2013;19:840-8.

53 Schwappach DL, Frank O, Buschmann U, et al. Effects of an educational patient safety campaign on patients' safety behaviours and adverse events. J Eval Clin Pract 2013;19:285-91.

54 Kripalani S, Roumie CL, Dalal AK, et al. Effect of a pharmacist intervention on clinically important medication errors after hospital discharge: a randomized trial. Ann Intern Med 2012;157:1-10. 